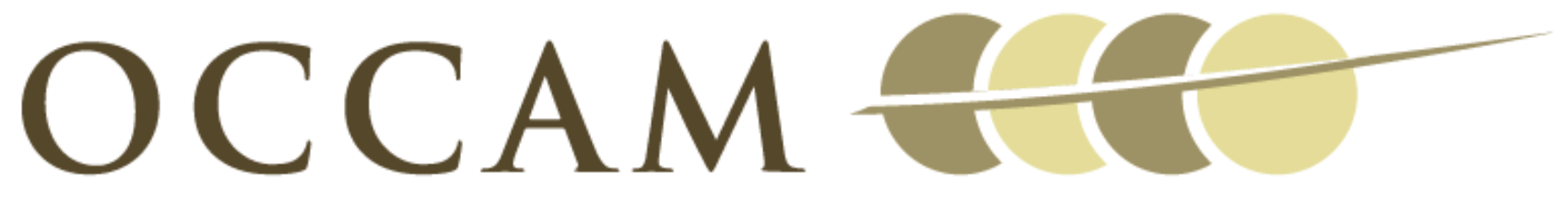

OXFORD CENTRE FOR COLLABORATIVE APPLIED MATHEMATICS

Report Number 10/02

Determining the equation of state of highly plasticised metals from boundary velocimetry

by

E.J. Hinch

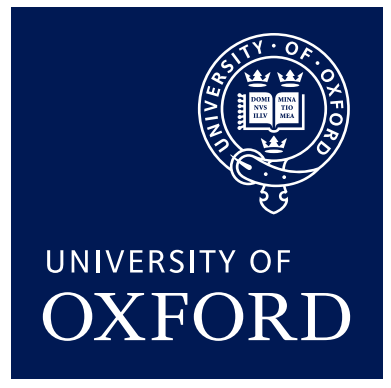

Oxford Centre for Collaborative Applied Mathematics Mathematical Institute

24 - 29 St Giles'

Oxford

OX1 3LB

England 



\title{
Determining the equation of state of highly plasticised metals from boundary velocimetry
}

Part II. An inverse problem or not

\section{E.J Hinch}

CMS-DAMTP, Cambridge University

\begin{abstract}
.
This is a follow-up paper to that of Ockendon, Ockendon and Platt, 2010. A more detailed derivation is provided, along with a numerical method which determines directly the full equation of state relating pressure to density. The issue of whether or not the problem is an inverse problem is discussed.
\end{abstract}

Keywords: Equation of state, hodograph, bootstrap numerical method

\section{Introduction}

As described by Ockendon, Ockendon and Platt, 2010, the problem is to determine the equation of state relating the pressure $p$ to the density $\rho$ from observations of the velocity as a function of time of one side of two samples of different thickness which are subjected to to same disturbance on the other side. The form of the disturbance is unknown. Experimental observations of the velocities, made at Sandia National Laboratories in collaboration with AWE (Rothman et al, 2005) and (Rothman and Maw, 2006), are plotted in figure 1. Some early small motion has been omitted and the data has been smoothed by cubic splines, following Ockendon, Ockendon and Platt, 2010. Note that in all cases the velocity increases monotonically in time, a feature required by the solution method.

The basic idea behind the determination of the equation of state is that the applied disturbance at one instant gives rise to the other sides of the two samples moving at the same velocity at different times. If reflected waves are ignored, the time difference in the arrival of the information gives the velocity of sound for that degree of compression. The problem is to compensate for the reflected waves. Rothman et al, 2005 and Rothman and Maw, 2006 tackled this problem by first assuming a polytropic form for $p(\rho)$ and then, by integrating numerically back and forth along the characteristics, found a first approximation to the effect of the reflected waves. This gave an improved estimate for the equation of state, which could be iterated further.

Ockendon, Ockendon and Platt, 2010 formulated the problem in the hodograph plane thereby linearising the equations and converting

(C) 2010 Kluwer Academic Publishers. Printed in the Netherlands. 


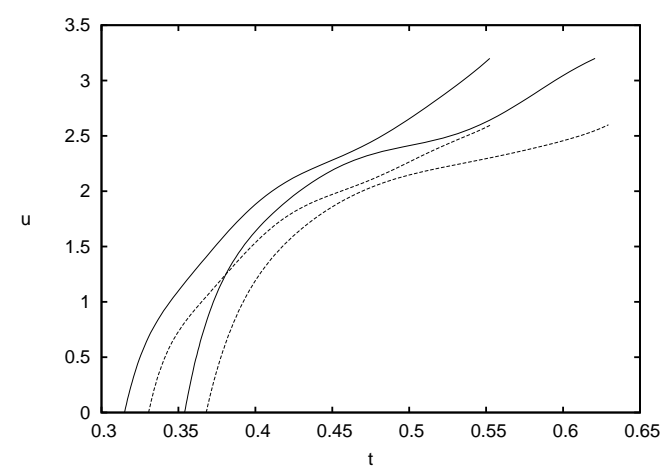

Figure 1. The velocities of the end in $\mathrm{km} \mathrm{s}^{-1}$ as a function of time in $\mu \mathrm{s}$. The two continuous curves are for experiment Pb753, and the two dashed curves for experiment $\mathrm{Pb} 770$. The upper of the two pairs is for the sample of length $0.2 \mathrm{~mm}$, and the lower for the sample of length $0.3 \mathrm{~mm}$.

the problem into an inverse problem, namely a wave equation with an unknown coefficient which depends on one of the independent variables. Treating the problem as an inverse problem, they chose to use a low-order Murnaghan parameterisation. They found the values of the parameters which gave the best fit to satisfying a certain boundary condition.

In this paper, the derivation of the hodograph transformation will be presented in fuller detail, describing several technical complications not mentioned in Ockendon, Ockendon and Platt, 2010. The problem will then be tackled by a bootstrap numerical method, which can determine the full equation of state. Finally the question of whether or not the problem is really an inverse problem will be discussed.

\section{Gas dynamics in Lagrangian coordinates}

It is assumed that at the very high pressures of Giga Pascals, which are orders of magnitude above the yield stress, the metal behaves as a compressible gas, increasing its density by over $50 \%$ during the experiment. For the wide samples used, a one-dimensional analysis is appropriate. It is convenient to use a Lagrangian coordinate $X$ in which the two moving ends become fixed material points, $X=0$ where the disturbance is applied and $X=L$ where the velocity is observed.

We start from the normal Eulerian equations of motion for gas dynamics

$$
\frac{\partial \rho}{\partial t}+u \frac{\partial \rho}{\partial x}+\rho \frac{\partial u}{\partial x}=0
$$




$$
\frac{\partial u}{\partial t}+u \frac{\partial u}{\partial x}+\frac{1}{\rho} \frac{\partial p}{\partial x}=0
$$

Consider a particle starting at $x=X$ at $t=0$ which moves with the flow

$$
\frac{d x}{d t}=u(x, t)
$$

The solution $x(X, t)$ gives the transformation between the Eulerian picture $(x, t)$ and the Lagrangian $(X, t)$. By the chain rule

$$
\begin{aligned}
\left(\frac{\partial}{\partial t}\right)_{X} & =\left(\frac{\partial}{\partial t}\right)_{x}+u\left(\frac{\partial}{\partial x}\right)_{t}, \\
\left(\frac{\partial}{\partial x}\right)_{t} & =\frac{\rho}{\rho_{0}}\left(\frac{\partial}{\partial X}\right)_{t},
\end{aligned}
$$

using the conservation of mass $\rho_{0} \delta X=\rho \delta x$. Hence the Lagrangian equations of motion are

$$
\begin{aligned}
& \frac{\partial \rho}{\partial t}+\frac{\rho^{2}}{\rho_{0}} \frac{\partial u}{\partial X}=0 \\
& \frac{\partial u}{\partial t}+\frac{1}{\rho_{0}} \frac{\partial p}{\partial X}=0
\end{aligned}
$$

The experiments are designed to have no shocks and only adiabatic changes. The flow will therefore be homentropic. The pressure will then be only a function of the density, i.e. the equation of state will be $p(\rho)$. The speed of sound $c(\rho)$ is given by

$$
c^{2}=d p / d \rho
$$

With the standard thermodynamic function $v(p)$ used in the Riemann invariants,

$$
v=\int_{0}^{p} \frac{d p}{\rho c}=\int_{\rho_{0}}^{\rho} \frac{c d \rho}{\rho},
$$

the governing equations (1) and (2) become

$$
\left(\frac{\partial}{\partial t} \pm \frac{\rho c}{\rho_{0}} \frac{\partial}{\partial X}\right)(u \pm v)=0
$$

i.e. the Riemann invariants $u \pm v$ are constant along characteristics $d X / d t= \pm \rho c / \rho_{0}$. Note that the Lagrangian speed of sound is

$$
c_{L}=\frac{\rho}{\rho_{0}} c,
$$

which measures the wave speed experienced by a material particle. 


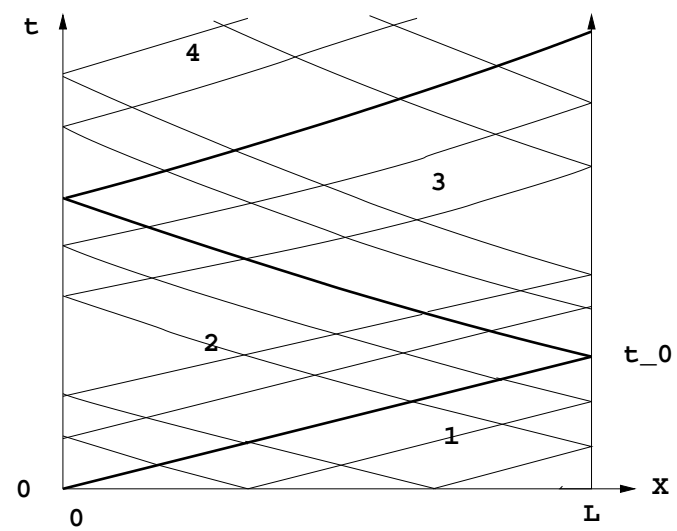

Figure 2. The Lagrangian domain, with regions: 1 at rest, 2 simple waves, 3 one reflection from right end, and 4 an additional reflection from left end.

In the Lagrangian picture, see figure 2, the left end of the sample is fixed at $X=0$ and the right end at $X=L$. Initial conditions of rest and zero pressure are applied throughout the sample so that

$$
u=0 \text { and } p=0 \text {, at } t=0 \text { for } 0 \leq X \leq L .
$$

Note that we have set the pressure to zero because the applied pressures at the left end are typically $10^{5}$ times the atmospheric pressure and $10^{3}$ times the yield stress, which may be more relevant. The boundary condition at the right end is thus

$$
p=0 \quad \text { at } \quad X=L \quad \text { for } \quad t \geq 0 .
$$

The boundary condition at the left end $X=0$ is unknown, although disturbances start at $t=0$.

With information propagating along characteristics, we deduce that region 1 in figure 2 will be at rest, $u=0=v$. In region 2 , there will be simple waves with $u=v$ taking information of the disturbances at the left end along straight characteristics. Region 3 will have waves reflected from the right end, and in region 4 these waves will be reflected one more time from the left end.

The experimental observations are of the velocity $u_{R}$ at the right end, $X=L$. The velocity will be zero up to $t=t_{0}$ when the first disturbance arrives from the left end. Our aim will be to calculate the information $u_{S W}$ arriving on the simple waves to the boundary between regions 2 and 3 , which will be the same information for the two samples of different thicknesses, because they have the same source at the left end. To find this information in these simple waves, we will effectively integrate back down the characteristics from the right end $X=L$ 
where the experimental observations are made. Integrating down these characteristics requires knowledge of the thermodynamic behaviour of the sample, in particular the speed of sound $c(\rho)$. The thermodynamic functions are not known a priori, and we seek them such that the information in the simple waves is the same for the two samples. The Riemann invariant propagated along the positive characteristic is $u+v$. At the right end it is equal to $u_{R}$ because $v=0$ there, while in the simple wave region 2 it is $2 u_{S W}$ because $u=v$ there. Hence for each positive characteristic

$$
u_{S W}=\frac{1}{2} u_{R},
$$

a result which we will use repeatedly.

\section{Hodograph transformation}

The Lagrangian governing equations (1) and (2) with dependent variables $u(X, t)$ and $\rho(X, t)$ are nonlinear in those dependent variables. We now make a transformation in which $u$ and $p$ (which is a direct function of $\rho$ ) become the independent variables, and the resulting equation is linear in a new dependent variable $A(u, p)$.

First we define the velocity potential $\phi(X, t)$ by

$$
\phi(X, t)=\int_{L}^{X} u\left(X^{\prime}, t\right) d X^{\prime}, \quad \text { so that } \quad u=\frac{\partial \phi}{\partial X},
$$

where the limit of integration has been chosen so that $\phi(L, t)=0$. Thus $\phi(X, t)=0$ throughout the region 1 of the rest state. The Lagrangian conservation of momentum (2) is then

$$
\frac{\partial p}{\partial X}=-\rho_{0} \frac{\partial u}{\partial t}=-\rho_{0} \frac{\partial^{2} \phi}{\partial X \partial t},
$$

so we can take

$$
p=-\rho_{0} \frac{\partial \phi}{\partial t} .
$$

Hence a useful alternative formula for the velocity potential is

$$
\phi(X, t)=-\frac{1}{\rho_{0}} \int_{0}^{t} p\left(X, t^{\prime}\right) d t^{\prime}
$$

For the hodograph transformation, we define the action $A(u, p)$ by

$$
A(u, p)=-\frac{p}{\rho_{0}} t+u X-\phi(X, t) .
$$


Then

$$
\begin{aligned}
d A & =-\frac{1}{\rho_{0}}(d p t+p d t)+d u X+u d X-\frac{\partial \phi}{\partial X} d X-\frac{\partial \phi}{\partial t} d t \\
& =-\frac{1}{\rho_{0}} t d p+X d u
\end{aligned}
$$

Hence using subscripts to denote differentiation

$$
A_{p}=-t / \rho_{0} \quad \text { and } \quad A_{u}=X .
$$

Differentiating these two with respect to $t$ at constant $X$ gives

$$
\begin{aligned}
& A_{p p} p_{t}+A_{p u} u_{t}=-1 / \rho_{0}, \\
& A_{u p} p_{t}+A_{u u} u_{t}=0 .
\end{aligned}
$$

So

$p_{t}=-A_{u u} / \rho_{0} D \quad$ and $\quad u_{t}=A_{p u} / \rho_{0} D \quad$ where $\quad D=\left(A_{p p} A_{u u}-A_{p u}^{2}\right)$

Similarly differentiating the two with respect to $X$ at constant $t$ gives

$$
p_{X}=-A_{p u} / D \quad \text { and } \quad u_{X}=A_{p p} / D .
$$

The relation between the two cross derivatives is an expression of the conservation of momentum (2).

We can now turn to the Lagrangian mass conservation equation (1). The first term is

$$
\frac{\partial \rho}{\partial t}=\frac{1}{c^{2}} \frac{\partial p}{\partial t}=-\frac{A_{u u}}{c^{2} \rho_{0} D}
$$

While the second terms is

$$
\frac{\rho^{2}}{\rho_{0}} \frac{\partial u}{\partial X}=\frac{\rho^{2} A_{p p}}{\rho_{0} D}
$$

The mass conservation (1) thus gives

$$
A_{u u}=\rho^{2} c^{2} A_{p p} .
$$

Note that the hodograph transformation has lead to a wave equation linear in $A$ with coefficients varying in the dependent variable $p$ through $\rho$ and $c$ depending on $p$.

The variation of the coefficients in the wave equation above makes the characteristics curved. We now make a further transformation from $p$ to $v$, with

$$
\frac{\partial}{\partial p}=\frac{1}{\rho c} \frac{\partial}{\partial v}
$$




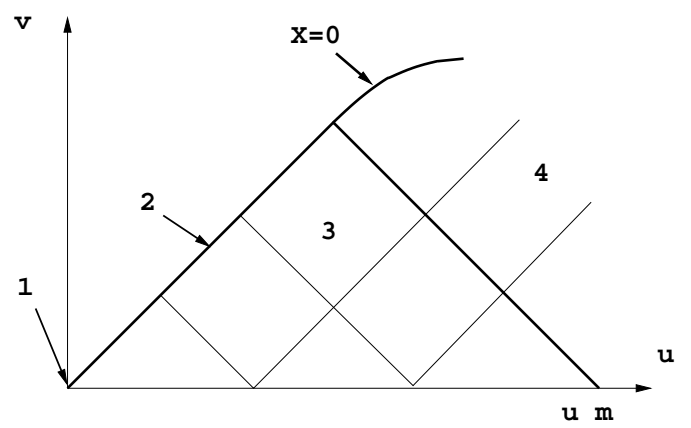

Figure 3. The hodograph plane with regions 1 at rest, 2 simple waves, 3 one reflection from right end, and 4 additional reflection from left end.

from the definition (3) of $v$. Equation (9) then becomes

$$
A_{u u}=A_{v v}+G(v) A_{v}
$$

where

$$
G=\rho^{2} c^{2} \frac{d}{d p}\left(\frac{1}{\rho c}\right)=-\frac{1}{c}\left(\frac{d c}{d v}+1\right)=-\frac{d}{d v} \log c_{L},
$$

on using (3). The wave equation (10) is linear and has straight characteristics. The characteristics are $u \pm v=$ const, which are the Riemann invariants of the original wave equation for $u$ and $\rho$. The information that is propagated by equation (10) is in the gradient of $A(u, v)$, which gives the position $X$ and time $t$ through equation (8). Thus the formation of a shock would be determined by two positions in the $u v$-plane having the same value of $A_{u}$ and $A_{p}=A_{v} / \rho c$.

A general difficulty with the hodograph transformation is that the location of the initial and boundary conditions must be found.

The initial condition of rest, $u=0$ and $v=0$, is mapped to the single point of origin, marked 1 in figure 3 . Note that the function $A(u, p)$ is singular at the origin, because the partial derivative $\partial A / \partial u=X$ takes all values in $[0, L]$ for the initial conditions.

The right end $X=L$ has $p=0$, so $v=0$, for the experimentally observed values of velocity $u$, and hence is part of the horizontal $u$-axis. The time of an observed velocity $t(u)$ gives the boundary condition on the normal derivative $\partial A / \partial v=-c_{0} t(u)$, where $c_{0}$ is the speed of sound in the rest state $v=0$. The value of $A$ follows from the definition (6) with $p=0, X=L$ and $\phi=0$, so $A=u L$. Collecting these conditions, we have boundary conditions in the hodograph plane for the wave equation (10)

$$
A=u L, \quad \frac{\partial A}{\partial v}=-c_{0} t(u) \quad \text { on } \quad v=0 .
$$


Note that there would be a problem for this boundary condition if the observed velocity of the end did not increase monotonically in time.

Each simple wave in figure 2 has $u=v$. Hence in the hodograph $u v$ plane, each wave becomes a single point along the straight line $v=u$, marked as 2 in figure 3 . Note that, as with the origin, the function $A(u, v)$ is therefore singular all along the line $v=u$, because the partial derivative $\partial A / \partial u=X$ takes a range of values. To avoid the singularities at the origin and along $v=u$, we need to restrict attention to strictly within region 3 of the first reflected waves.

The waves in figure 2 which have been reflected from the right end have $u>v$ and hence lie between the horizontal $u$-axis and the simple waves on $v=u$, and are marked as region 3 in figure 3 . Figure 2 shows the relationship (4) between the value of $u$ in the initial conditions on $v=0$ and that in the simple waves follows simply from the straight line characteristic $u+v=$ const.

The left end is at $\partial A / \partial u=X=0$. The location in the hodograph plane of this boundary must be determined from the solution of the wave equation (10). Part of the boundary will lie in the simple waves. When the boundary leaves the simple waves, there are waves which have reflected off both the right and then the left end, marked as region 4 in figure 3 . The last reflected wave to become a simple wave rather than first arrive at the left end, i.e. the boundary between regions 3 and 4 in figure 3 , is labelled by the maximum value of $u$ on the axis $v=0$ as $u_{\mathrm{m}}$.

\section{The difference problem}

If we knew the equation of state $p(\rho)$, then we would know the thermodynamic function $G(v)$ in the wave equation (10) for A. It would then be possible to integrate directly this wave equation forward from the boundary conditions (12) to find $A(u, v)$ for the simple waves on $v=u$, region 2 in figure 3 . However we do not know a priori the equation of state; it is our task to find it. Instead we know for two samples of different lengths $L_{1}$ and $L_{2}$ the arrival times, $t_{1}\left(u_{R}\right)$ and $t_{2}\left(u_{R}\right)$, of the velocity $u_{R}$ of the right end. These two arrival times come from the same simple wave, the one with $v=u=\frac{1}{2} u_{R}$ from equation (4).

Let $A_{1}$ and $A_{2}$ be the solutions of the wave equation (10) with boundary conditions (12) corresponding to the two samples with $L_{1}$, $t_{1}(u)$ and $L_{2}, t_{2}(u)$. Consider the difference

$$
A(u, v)=A_{1}(u, v)-A_{2}(u, v) .
$$


Because the wave equation and boundary conditions are linear, the difference $A$ will satisfy the same equation (10) and will satisfy boundary conditions

$$
A=u \Delta L, \quad \frac{\partial A}{\partial v}=-c_{0} \Delta t(u) \quad \text { on } \quad v=0,
$$

where

$$
\Delta L=L_{1}-L_{2} \quad \text { and } \quad \Delta t(u)=t_{1}(u)-t_{2}(u) .
$$

For the two samples, the two simple waves with the same values of $v=u$ have the same source at the left end. Thus using the formula for the velocity potential (5), we deduce at the left end $X=0$ the two simple waves have the same values of $\phi$ and $p$, and hence the same value of $A$ by (6). Along a simple wave both $p$ and $u$ are constant, so that by (7) the common value of $A$ does not change along the two simple waves. Hence we deduce that at the boundary between the simple waves in region 2 and the reflected waves in region 3

$$
A=A_{1}-A_{2}=0 \quad \text { on } \quad v=u .
$$

The aim is now to find the thermodynamic function $G(v)$ so that the difference solution $A(u, v)$ of the wave equation (10) satisfying difference boundary conditions (13) vanishes at the simple waves (14).

Now on the simple waves $v=u$, the difference solution vanishes, $A=0$. Differentiating this along the line $v=u$ (strictly just within region 3 of the first reflected waves), we have on $v=u$

$$
\frac{\partial A}{\partial u}+\frac{\partial A}{\partial v}=0 .
$$

Now all the way along $v=0, \partial A / \partial u=\Delta L$, while at $v=u=0$, the boundary condition gives $\partial A / \partial v=-c_{0} \Delta t(0)$. Hence the above equation gives the speed of sound in the rest state is given in terms of the experimental data

$$
c_{0}=\frac{\Delta L}{\Delta t(0)} .
$$

Thus the first simple wave travels at speed $c_{0}$ because it travels through material at rest encountering no reflected wave. Further derivatives along the line $v=u$ can be made to obtain local information about $G(v)$. For example, the second derivative gives

$$
G(0)=-\left.2 \frac{1}{\Delta t} \frac{d \Delta t}{d u}\right|_{u=0} .
$$

In practice, however, the experimental data is too noisy to be differentiated to obtain such local information reliably. 


\section{Numerical bootstrap method}

In this section, we will solve the wave equation for $A(u, v)$ numerically, working away from the boundary $v=0$ to a particular value of $v$. This solution, together with condition (14) at the next value of $v$, will give $G$ at $v$. This value of $G$ can then be used in the wave equation (10) to find the solution $A(u, v)$ at the next value of $v$. Thus we can 'bootstrap' our way to find the full solution of the wave equation in the region 3 of figure 3 , along with the thermodynamic function $G(v)$.

The wave equation is tackled by second-order finite differences using the same equal spacing of the grid in the two directions, i.e. we set $\delta v=\delta u$. Because the wave equation (10) has straight characteristics $v= \pm u+$ const., the characteristics go exactly through the grid points, and so the numerical solution would be exact if $G$ were zero and the boundary conditions were treated exactly.

To implement the derivative boundary condition with second-order accuracy, we set

$$
A(u, \delta v)=A(u, 0)+\delta v A_{v}(u, 0)+\frac{1}{2} \delta v^{2} A_{v v}(u, 0) .
$$

Because $A(u, 0)=u \Delta L$, the wave equation (10) gives at $v=0$

$$
A_{v v}=-G(0) A_{v}
$$

while the derivative boundary condition is

$$
A_{v}=-c_{0} \Delta t(u) \text {. }
$$

Hence, to second order

$$
A(u, \delta v)=u \Delta L-\delta v c_{0} \Delta t(u)\left(1-\frac{1}{2} \delta v G(0)\right) .
$$

Applying this to the simple wave at $u=\delta v$ where $A(u, \delta v)=0$ gives the first value of the function $G(v)$

$$
G(0)=\frac{2}{\delta v} \frac{\Delta t(\delta v)-\Delta t(0)}{\Delta t(\delta v)}
$$

In the finite-difference solution, we write

$$
A_{i j}=A(u=i \delta v, v=j \delta v) \text { and } G_{j}=G(v=j \delta v) .
$$

The wave equation at the $i j$ grid point, with $i>j$ in order to be in region 3 of the reflected waves, then becomes with second-order central differencing

$$
A_{i j+1}=\frac{A_{i+1 j}+A_{i-1 j}-A_{i j-1}\left(1-\frac{1}{2} \delta v G_{j}\right)}{1+\frac{1}{2} \delta v G_{j}}
$$




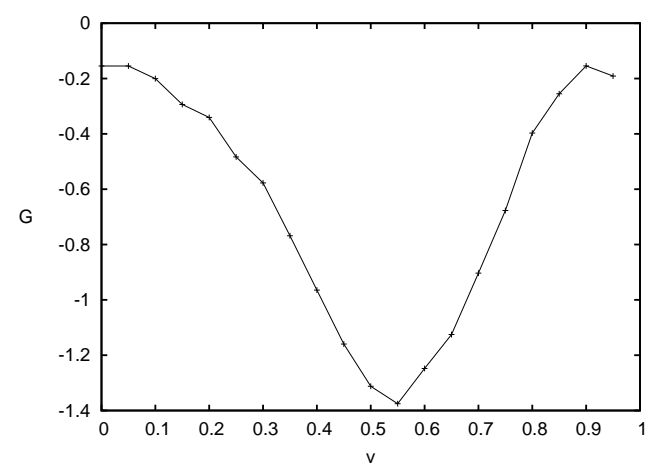

Figure 4. The thermodynamic function $G$ in $\mathrm{m}^{-1} \mathrm{~s}$ as a function of $v$ in $\mathrm{ms}^{-1}$ for the experiment $\mathrm{Pb} 753$ using 40 slices of velocity.

Applying this to the point the first point $i=j+1$ inside region 3 , we have

$$
G_{j}=\frac{2}{\delta v} \frac{A_{j+1 j-1}-A_{j+2 j}}{A_{j+1 j-1}}
$$

using the simple wave property $A_{i j+1}=0=A_{i-1 j}$ when $i=j+1$.

The 'bootstrap' approach is then the following. From previous stages, one has the solution of the wave equation $A_{i j}$ for $i \geq j$ up to $j=J$, and thermodynamic function $G_{j}$ up to $j=J-1$. Equation (19) then gives $G_{j}$ for $j=J$. With this value of $G_{J}$, equation (18) gives $A_{i j}$ for $j=J+1$. This procedure is started at $J=1$ using $A_{i 0}$ from (13a) and $A_{i 1}$ from (16).

Figure 4 gives the numerical result for $G(v)$ using 40 equally spaced values of the observed velocity $u$ of the right end in one experiment $\mathrm{Pb753}$. The quality of the result is poor because the process of extracting $G(v)$ is equivalent to a numerical differentiation of the noisy experimental data.

Using this result for $G(v)$, we can then calculate the function $A_{1}(u, v)$ for the sample of length $L_{1}$. The value of its derivative $\partial A_{1} / \partial u$ along $u=v$, evaluated numerically from $u \geq v$ to second order accuracy by extrapolation, gives the location $X$ in the Lagrangian picture of the boundary between the reflected and simple waves. This location is plotted in figure 5 as a function of the value of $u=v$ of the simple wave. The low velocities correspond the the early arriving waves which cease being simple waves just before $X=L_{1}=0.2 \mathrm{~mm}$. At large velocities $X$ is predicted to become negative. This erroneous prediction is a consequence ignoring the second reflection from the left end. As we do not know how to take into account the second reflection, we must limit our use of the experimental data to $v \leq 1.06$ for experiment $\mathrm{Pb} 753$, and $v \leq 0.97$ for experiment $\mathrm{Pb} 770$. These values of $v$ are for 


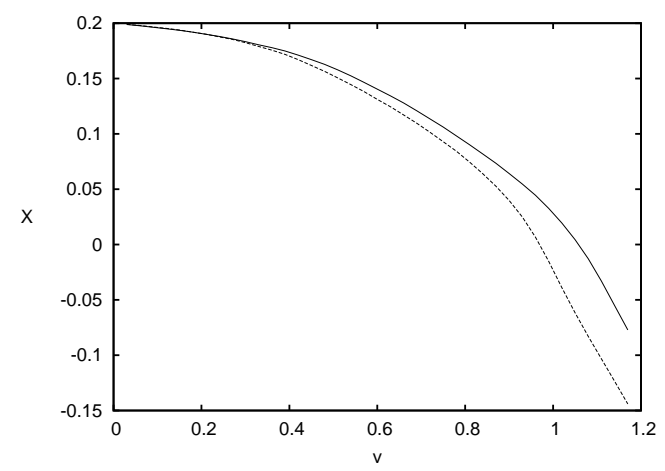

Figure 5. The Lagrangian location $X$ in $\mathrm{mm}$ as a function of $u=v$ in $\mathrm{km} \mathrm{s}^{-1}$ of the boundary between the reflected and simple waves. The continuous curve is for experiment $\mathrm{Pb} 753$, the dashed curve for experiment $\mathrm{Pb} 770$.

the simple wave where $u=v$. Using (4), the maximum values of the observed velocities of right end are then $u_{\mathrm{m}}=2.12$ and 1.94. Looking back to figure 1 for the experimentally observed velocities at the right ends as a function of time, one can observe kinks on the curves at these $u_{\mathrm{m}}$, now understood as the arrival of second reflections. In the results presented below, we only use experimental data less than 2.1 and 1.9 respectively.

The numerical method above delivers the thermodynamic function $G(v)$ defined in (11). From this we now evaluate the equation of state $p(\rho)$. We integrate the following ordinary differential equations

$$
\frac{d c}{d v}=-1-c G, \quad \frac{d p}{d v}=\rho c, \quad \text { and } \quad \frac{d \rho}{d v}=\frac{\rho}{c}
$$

with initial conditions

$$
c(0)=c_{0}, \quad p(0)=0 \quad \text { and } \quad \rho(0)=\rho_{0} .
$$

Figure 6 gives the (Eulerian) velocity of sound $c$ as a function of the density $\rho$ for the experiment $\mathrm{Pb} 753$. Note that the curve is an integral of $G(v)$ and thus much smoother. Note the change of numerical resolution from 40 to 80 equally spaced velocities does not visibly change the predicted velocity of sound.

The corresponding equation of state $p(\rho)$ is plotted in figure 7 , and this is an even smoother curve since it involves another integration. Results are given for the two experiments, $\mathrm{Pb} 753$ and $\mathrm{Pb} 770$. The are moderately close. Both curves appear to be two nearly straight lines with a change of slope around $\rho=1410^{3} \mathrm{~kg} \mathrm{~m}^{-3}$, which is roughly where the speed of sound in figure 6 changes from 2.6 to $3.4 \mathrm{~km} \mathrm{~s}^{-1}$. Rothman et al, 2005 suggest in their figure 5 that there is a phase transition from $f c c$ to $h c p$ around $13 \mathrm{G} \mathrm{Pa}$. 


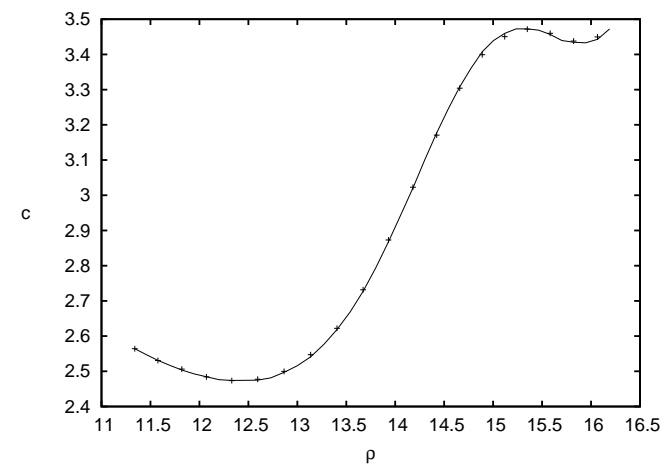

Figure 6. Velocity of sound in $\mathrm{km} \mathrm{s}^{-1}$ for experiment $\mathrm{Pb} 753$ as a function of density in $10^{3} \mathrm{~kg} \mathrm{~m}^{-3}$. The continuous curve uses 80 equally spaced velocities, while the points use only 40 .

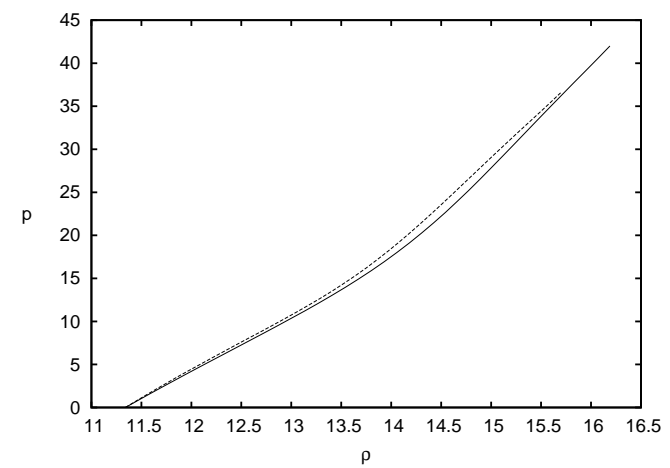

Figure 7. Equation of state, pressure in $\mathrm{GPa}$ as a function of density in $10^{3} \mathrm{~kg} \mathrm{~m}^{-3}$. The continuous curve is the experiment $\mathrm{Pb} 753$ and the dashed curve for experiment $\mathrm{Pb} 770$.

Ockendon, Ockendon and Platt, 2010 chose to represent the equation of state by the low-order parameterisation of the Murnaghan model

$$
p=p_{0}\left(\left(\frac{\rho}{\rho_{0}}\right)^{\gamma}-1\right)
$$

Table I. Murnaghan fit (20) for Pb753 with $\rho_{0}=11.34$.

\begin{tabular}{cccc}
\hline Range of $\rho$ & $p_{0}$ & $\gamma$ & Rms residual \\
\hline $11.34-16.0$ & 14.5 & 3.81 & 0.44 \\
$11.34-13.5$ & 77.1 & 0.93 & 0.0022 \\
$13.00-14.5$ & 19.6 & 3.05 & 0.020 \\
$14.00-16.0$ & 12.2 & 4.24 & 0.032 \\
\hline
\end{tabular}


where $p_{0}, \rho_{0}$ and $\gamma$ are all constants. It is possible to fit the numerical results for the equation of state to this model. Table I gives the best-fit values of the parameters $p_{0}$ and $\gamma$ for different ranges of variation of density. The base density $\rho_{0}$ is held fixed at 11.34. Fitting over the whole range, the best values are $p_{0}=14.5$ and $\gamma=3.81$. The rms residual error of the fit to the equation of state is a relatively large 0.44 . The rms error is significantly smaller if the fit is made to the two sub-ranges before and after $\rho=13.7$, reflecting the feature that figure 7 is more like two straight lines joined together rather than a single power-law. In other words, the Murnaghan model may not be appropriate model for the physics. Note that Ockendon, Ockendon and Platt, 2010 minimised the error in satisfying a certain boundary condition, and could not estimate of the rms residual error in the fit to the equation of state.

\section{Conclusions}

The use of the hodograph transformation turns the difficult original nonlinear governing equations (1) and (2) into a linear wave equation (9), and after the further transform into a linear wave equation (10) with straight characteristics. This is a significant improvement. Because the equation is linear, the solution can be constructed by linear supposition through the use of Riemann functions, as used by Ockendon, Ockendon and Platt, 2010. The original nonlinear problem cannot be tackled in this way. A technical difficulty that must be mastered is tracking down the initial and boundary conditions in the transform plane. Moreover, the method needs the entropy to be constant in time and space, and so shocks must not occur. A another small problem is that the further transformation which straightens the characteristics introduces in $G(v)$ an additional differentiation of the experimental data.

On first inspection, the problem to solve the wave equation (10) subject to boundary conditions (13) and (14) appears to be a classical inverse problem. The governing partial differential equation contains an unknown function of position, and there are too many boundary equations. The inverse problem is to find the unknown function so that the excessive number of boundary conditions can be satisfied. This is the reason that Ockendon, Ockendon and Platt, 2010 tackled the problem with a standard inverse approach.

A difficulty with inverse problems is that they are notoriously illconditioned; large variations in the unknown function can make undetectable changes when fitting the experiment data. For this reason only a low-order Murnaghan parameterisation was used by Ockendon, 
Ockendon and Platt, 2010, avoiding the use of more sophisticated 'regularisations'. The origin of this ill-conditionedness is the averaging behaviour of elliptic and parabolic partial differential equations. For example, significant variations in the thermal conductivity in the interior will have a small effect on the observables at the boundary if the variations have a sufficiently small spatial scale. An extreme example would be for the thermal conductivity to vanish on the boundary of an interior region, so that the exterior would be totally blind to all variation within that region.

The problem tackled in this paper is different. It is governed by a hyperbolic equation, in which each simple wave propagates information without change along one characteristic without communicating with an adjacent characteristic. Also in the original form of equations (1) and (2), the unknown is not a function of position but a function of the dependent variables, i.e. there is an unknown nonlinearity which has no fine scale variations in space. One can obtain a good estimate of the velocity of sound, with only a $25 \%$ error to $v=0.5 \mathrm{~m} \mathrm{~s}^{-1}$, by ignoring the reflected waves and just looking at the difference in positions for the two samples divided by the differences in arrival times of an endvelocity. In this simplified estimate, relative changes in the observable boundary data are precisely equal to the relative changes in the speed of sound, and so there is none of the classical averaging which leads to ill-conditionedness. When the reflected waves are taken into account, there is still the direct association of the speed and the corresponding arrival times, with a subtraction of a small integral over previously determined speeds of sound. Because the integral is typically only a $25 \%$ subtraction, the good conditionedness is not lost.

The numerical bootstrap approach used in section 5 is a direct forward method, demonstrating that the problem is unexpectedly not a classical inverse problem despite initial appearances. There is an interesting question of how to decide whether a problem is an unavoidable inverse problem or not.

The bootstrap approach can be used to tackle the original problem in the Lagrangian plane. Details are given in the appendix.

The results for the equation of state are found not to follow the Murnaghan model well, because the best-fit vales of its parameters change significantly when the fitting range is changed. This difficulty may be due to a change of phase. A small problem with the global approach of Ockendon, Ockendon and Platt, 2010 is that one cannot tell how large the residual errors are in the fit to the equation of state from the size of the global error in satisfying a boundary condition. 


\section{Appendix}

In section 5, a numerical method was presented for solving the wave equation in the $u v$-hodograph plane. The same bootstrap idea can be applied to integrating along the characteristics in the Lagrangian $X t$ plane.

Rothman and Maw, 2006 have developed a method of integrating along the characteristics in the Lagrangian plane. They first assume an approximate equation of state. This is used to integrate down the positive characteristics from the right end to the simple waves. Comparing the positions and times of the two samples for a simple wave with the same $u_{R}$, they can evaluate the Lagrangian sound speed $c_{L}(v)$. Integrating this yields an improved approximation to the equation of state. The whole process can then be repeated until a converged equation of state is obtained.

In the bootstrap approach, one integrates down the positive characteristics one at a time, starting at $u_{R}=0$ and moving to increasing end-velocities. In the integration, each new characteristic only needs values of the speed of sound which have already been determined, those for $v=0$ to just below $v=\frac{1}{2} u_{R}$. On arriving at the region of simple waves, the difference between the two samples of the positions and times produces the speed of sound for the next higher value of $v=\frac{1}{2} u_{R}$.

In a little more detail, let the $\left(X_{i j}, t_{i j}\right)$ be the intersection in the Lagrangian $X t$-plane of the positive characteristic through the right end when it is moving at $2 i \delta v$ and the negative characteristic through the right end when it is moving at $2 j \delta v(j \leq i)$. Note that here we need the velocity of the end to increase monotonically in time. The Riemann invariants for these two characteristics, which can be evaluated at the right end where $v=0$, give for this intersection $u=(i+j) \delta v$ and $v=(i-j) \delta v$. Write the speed of sound $c(v=i \delta v)=c_{i}$. The $i$-th positive characteristic is started at the right end, $j=i$, where $X_{i i}=L$ and $t_{i i}$ is equal to the experimental time when the end has velocity $2 i \delta v$. The integration down the positive characteristic will be stopped at the boundary with the simple waves where $j=0$. To move along the positive characteristic from $X_{i j+1}$ to $X_{i j}$ with $j \geq 1$, one solves for the intersection of two short segments of straight lines, one for the positive characteristic from $X_{i j+1}$ with the slope $\frac{1}{2}\left(c_{i-j}+c_{i-j-1}\right)$, and one for the negative characteristic through $X_{i-1 j}$ with the opposite sign of the same slope. The values of the speed of sound $c_{0}$ to $c_{i-1}$ are known from integrating down previous positive characteristics. Note that the use of equal increments in end velocity mean that no interpolation is needed in the above evaluation of the speed of sound at the intersections. Use of the average of the slopes between the beginning and end of the short 
segments gives a numerical method with second-order accuracy. For the last step down the characteristic to the simple wave, $j=0$, one must use the cruder first-order slope of $c_{i-1}$, because $c_{i}$ is not yet known. However the net error of a single first-order step is the same as many steps of second-order. Finally the speed of sound $c_{i}$ is evaluated from the difference between the two samples in positions $X_{i 0}$ divided by the difference in times $t_{i 0}$. This completes the bootstrap.

\section{Acknowledgements}

This work is based on work supported by Award No. KUK-C1-01304, made by the King Abdullah University of Science and Technology (KAUST). I would like to thank H. Ockendon, J.R. Ockendon and J. Platt for bringing the problem to my attention, and to thank S.D. Rothman and C.M. Robinson for providing the experimental data.

I am pleased to be able to contribute to this issue dedicated to Normam Riley, a good friend over many years.

\section{References}

Ockendon, H, Ockendon, JR, Platt, J (2010) Determining the Equation of State of Highly Plasticised Metals from Boundary Velocimetry. Submitted to J Engg Maths

Rothman, SD, Davis, J-P, Maw, J, Robinson, CM, Parker, K, Palmer, KJ, Measurement of the Principal Isentropes of Lead and Lead-Antimony Alloy to 400 kbar by Quasi-Isentropic Compression. Journal of Physics D: Applied Physics 38: $733-740$

Rothman, SD, Maw, J Characteristics analysis of isentropic compression experiments. J Phys IV France 134: 745-750

Address for Offprints: CMS-DAMTP, Cambridge University, Wilberforce Road, Cambridge, CB3 0WA. 
report21b.tex; 22/02/2010; 15:53; p.18 



\section{RECENT REPORTS}

26/09 Functional differential equations arising in cell-growth Wake

Begg

27/09 A Cell Growth Model Revisited Derfel

van Brunt

Wake

28/09 Quasi-steady state reduction of molecular motor-based models of Newby directed intermittent search

Bressloff

29/09 All-at-once preconditioning in PDE-constrained optimization Rees

Stoll

Wathen

30/09 An hp-Local Discontinuous Galerkin method for Parabolic Pani Integro-Differential Equations $\quad$ Yadav

31/09 Stochastic neural field theory and the system-size expansion Bressloff

32/09 A Hamiltonian Krylov-Schur-type method based on the symplec- Benner tic Lanczos process

Faßbender

Stoll

33/09 Nematic liquid crystals : from Maier-Saupe to a continuum theory Ball

Majumdar

34/09 Tangent unit-vector fields: nonabelian homotopy invariants and Majumdar the Dirichlet energy

Robbins

Zyskin

35/09 A metabolite-sensitive, thermodynamically-constrained model of Tran cardiac cross-bridge cycling: Implications for force development Smith during ischemia

Loiselle

Crampin

36/09 Modelling bacterial behaviour close to a no-slip plane boundary: Shum the influence of bacterial geometry

Gaffney

Smith

Goswami

Pani imation to a second order linear parabolic initial and boundary value problem with nonsmooth initial data

38/09 Optimal L2 estimates for semidiscrete Galerkin methods for parabolic integro-differential equations with nonsmooth data

Goswami

Pani

Yadav

39/09 Spatially structured oscillations in a two-dimensional excitatory

Kilpatrick neuronal network with synaptic depression

Bressloff

40/09 Stationary bumps in a piecewise smooth neural field model with synaptic depression

Kilpatrick

Bressloff 
41/09 Homogenization for advection-diffusion in a perforated domain

Haynes

Hoang

Norris

Zygalakis

42/09 Fast stochastic simulation of biochemical reaction systems by al-

Melykuti ternative formulations of the Chemical Langevin Equation

Burrage

Zygalakis

43/09 Pseudoreplication invalidates the results of many neuroscientific

Lazic studies

44/09 Cardiac cell modelling: Observations from the heart of the cardiac physiome project

45/09 A Hybrid Radial Basis Function - Pseudospectral Method for Thermal Convection in a 3-D Spherical Shell

Wright

Flyer

46/09 Refining self-propelled particle models for collective behaviour

Yates

Baker

Erban

Maini

47/09 Stochastic Partial Differential Equations as priors in ensemble methods for solving inverse problems

Potsepaev

Farmer

Aziz

48/09 DifFUZZY: A fuzzy spectral clustering algorithm for complex data

Cominetti et al. sets

01/10 Fluctuations and instability in sedimentation

Guazzelli

Hinch

Copies of these, and any other OCCAM reports can be obtained from:

Oxford Centre for Collaborative Applied Mathematics Mathematical Institute

24 - 29 St Giles'

Oxford

OX1 3LB

England

www.maths.ox.ac.uk/occam 